\title{
Linear system construction of multilateration based on error propagation estimation
}

\author{
Yanjun $\mathrm{Hu}^{1,3}$, Lei Zhang ${ }^{1,3}$, Li Gao ${ }^{2^{*}}$, Xiaoping $\mathrm{Ma}^{3}$ and Enjie Ding ${ }^{1}$
}

\begin{abstract}
Iterative localization algorithms are critical part in the control of mobile autonomous robots because they feed fundamental position information to the robots. In a harsh unknown environment, the estimation of environmental noise is hardly obtained during the movement of the robots. It means that the state-of-the-art methods, which increase localization accuracy using error management, are unsuitable. In this paper, we deduced an upper bound of the localization error without knowing the precise model of environment noise when the anchor nodes have position errors. Utilizing the minimum upper bound, we can construct an optimal localization linear system of iterative localization algorithms based on least square. An algorithm of generating localization linear system is proposed by using the minimum upper bound. The algorithm reduces the impact of the shortage of environmental information on localization error propagation. Our simulation results show that the algorithm is insensitive to noise and can improve the localization accuracy by constructing a proper localization linear system with a high probability.
\end{abstract}

Keywords: Iterative localization, Error propagation, Upper bound, Orthogonally invariant norms

\section{Introduction}

Indoor iterative localization algorithm of autonomous robots is an active subject because of the environmental complexity. The coordinates of autonomous robots are the fundamental parameters of robot control [1]. Due to the absence of Global Positioning System signals in an indoor-like environment, the autonomous robots need an iterative localization algorithm to provide their position information. Laser, sonar, infrared, visual sensors, or some combinations of these methods are used to locate the robots [2]. However, those technologies may fail in some harsh environments, such as a firing building which is dusty, smoky, and dark.

Iterative localization based on Received Signal Strength (RSS) is a suitable option to provide the position information in a harsh scenario [3]. Indoor iterative localization based RSS is exploited in ranging-based techniques, which maps the distance by a measurement of RSS, see

\footnotetext{
* Correspondence: gaoli@jsnu.edu.cn
}

${ }^{2}$ School of Electrical Engineering and Automation, Jiangsu Normal University, Xuzhou, People's Republic of China

Full list of author information is available at the end of the article e.g., [4-6]. Since the measurement noise is inevitable in the practical localization system, algorithms are proposed to improve the localization accuracy. In those algorithms, the key component of decreasing localization error is to estimate the measurement noise. And, those algorithms work effectively if the noise is estimated precisely [7]. However, in a harsh scenario, the noise estimation is almost impossible to be achieved because of the insufficiency on measurement noise caused by the robots mobility [8]. A new strategy of improving localization accuracy is needed to solve the problem. Actually, localization accuracy is influenced by the construction of localization linear system (LLS) when least square is used to calculate the position of the unknown node. Therefore, we can decrease localization error by constructing proper LLS. In this paper, through the studying of upper bound of localization error of LLS, an upper bound of error propagation in the localization is proposed. And, an algorithm which can be used to improve the localization accuracy without environmental noise estimation, by utilizing the upper bound to generate an optimum LLS. 
The paper is organized as following: In Section 2, related works are introduced. In Section 3, the key step of iterative localization is briefly described to introduce the symbols. In Section 4, after introducing the orthogonally invariant norms, it is given that the error upper bound of localization using anchors with localization error. And, an algorithm of improving localization accuracy is proposed by constructing optimum LLS which uses the minimum upper bound. In Section 5, the random and efficient of algorithm are verified by simulations.

\section{Related work}

The iterative localization algorithm is a distributed, infrastructure-free positioning algorithm to calculate nodes' positions in the ad hoc networks $[9,10]$, which is a "spreading" process of node information. The process consists of three processes, which are node registry, neighbor selection, and update criterion [11].

The main difference between the iterative localization and the common localization is that the coordinates of located nodes are used or not to calculate the unknown nodes. In iterative localization, there exists unknown nodes that will use the nodes' coordinates which is calculated with localization error. It means that anchor node coordinates may or may not have errors in a coordinate calculation process. To distinguish the anchor nodes with position error from those nodes with precise coordinates, the anchor nodes without coordinate errors are named origin-anchors, while the others are called pseudo-anchors. Based on the notions, a typical iterative localization algorithm carries out in the following steps:

- Initiating nodes: Each node in the ad hoc network initiates its coordinate and the coordinate's errors.

- Selecting origin-anchors: Three or more nodes are selected as origin-anchors, whose coordinate errors are considered as zero. Then, a relative coordinate system is built by using those nodes.

- Generating pseudo-anchors: An unknown node selects at least three located nodes from the neighbor nodes set to calculate its position. After the node is located, this node is updated as a pseudo-anchor.

In this perspective, an iterative localization algorithm is a process of transforming the nodes into the pseudo-anchors.

Obviously, the localization accuracy is influenced by the last two steps. The method of selecting origin-anchors is studied, such as choosing the nodes with maximum density factor [9]. Consider the executing time, the process of pseudo-anchor generation will be executed more frequently than the process of origin-anchor selection. Therefore, improving the localization accuracy in the step of generating pseudo-anchors will significantly decrease the localization errors of all pseudo-anchors. To meet the requirement of improving localization accuracy, physical methods and cyber methods can be used.

In particular, physical methods are based on the idea that the less measurement error is the less localization error is. It improves localization accuracy by using more sensitive sensors. For example, passive broadband harmonic nonlinear transmission-line tags were used to measure the distance of two nodes [12, 13] or the distance was estimated by using the channel state information [14].

Meanwhile, under the constrains of the measurement accuracy limitation in the physical methods, cyber method is to design algorithms for finding an optimal position estimation of the unknown node. Multilateration based on Least-Squares (MLS) [15] is one widely used cyber method. Also, the localization error of MLS was studied to improve the localization accuracy [16]. Cramer Rao Bound is used to calculate the localization error bound [17-19], in which probability density function of noise is needed to calculate Fisher information matrix. And, localization accuracy was characterized by using a noise covariance bound when anchor nodes have location uncertainty [20]. To calculate localization error accumulating during an iterative process, the mean of localization error was given in the literature [11]. All those literatures assumed that the probability density function or covariance of noise is known. However, according to the description above, the assumption is not always satisfied in a harsh scenario. The peculiarity of localization in an anonymous environment is noticed [21]. But, the literature is focused on converting the RSS into the distance when little information on the radio propagation model is provided. It is still not studied that how to improve localization accuracy in the scenario of the insufficiency on measurement noise caused by the robots mobility. $\mathrm{Xu}$ et al. [22, 23] proposed a crowdsourcingbased framework for processing mobile information and have been proved to be a high accuracy and efficiency.

\section{Iterative localization based on least squares}

To introduce the notions and symbols used in the following contents, here, we briefly describe the process of MLS.

Let $\boldsymbol{x}=(x, y)$ represents the coordinate of an unknown node located based on an anchor nodes set $\left\{\boldsymbol{x}_{i}=\left(x_{i}, y_{i}\right) \mid i=\right.$ $1, \cdots, n\}$ where $n$ is the cardinality of the anchor nodes set, and \|\|$_{2}$ is the Euclidean norm. Localization based least squares performs as following:

First, the algorithm collects the measurement data, which is

$$
\left\|\boldsymbol{x}-\boldsymbol{x}_{i}\right\|_{2}=\hat{d}_{i} \quad(i=1, \cdots, n)
$$

where $\hat{d}_{i}$ denotes the measurement distance between the $i$ th anchor node and the unknown node. Squaring both sides of (1), a constraint is obtained 


$$
\|\boldsymbol{x}\|_{2}^{2}+\left\|\boldsymbol{x}_{i}\right\|_{2}^{2}-2 \boldsymbol{x}_{i}^{\mathrm{T}} \boldsymbol{x}=\hat{d}_{i}^{2} \quad(i=1, \cdots, n)
$$

It is obvious that there are $n$ constraints in a localization system.

Then, $i$ th anchor node is selected as the benchmarkanchor-node (BAN). Subtracting the $i$ th reference from all other constraints, we have:

$$
2\left(\boldsymbol{x}_{k}-\boldsymbol{x}_{i}\right) \boldsymbol{x}^{\mathrm{T}}=\left\|\boldsymbol{x}_{k}\right\|_{2}^{2}+\hat{d}_{k}^{2}-\left(\left\|\boldsymbol{x}_{i}\right\|_{2}^{2}-\hat{d}_{i}^{2}\right)
$$

where $k \neq i ; k=1, \cdots, n$. After that, we have a localization linear system (LLS) with $n-1$ equations:

$$
A_{i} \boldsymbol{x}^{\mathrm{T}}=\boldsymbol{b}_{i}
$$

The subscript $i$ of $A_{i}$ and $\boldsymbol{b}_{i}$ emphasizes that the $A$ and $\boldsymbol{b}$ are generated in the case of choosing $i$ th anchor node as BAN.

Finally, using the method of least-squares, the solution $\hat{\boldsymbol{x}}$ in (4), which is the estimated coordinate of the unknown node, is obtained

$$
\hat{\boldsymbol{x}}=\left(A_{i}^{\mathrm{T}} A_{i}\right)^{-1} A_{i}^{\mathrm{T}} \boldsymbol{b}_{i}
$$

The estimation coordinate of pseudo-anchor always deviates its physical coordinate since the measurement error exists. The literatures introduced in Section 2 have studied the methods of improving localization accuracy based on measurement error estimation. Unfortunately, as it is discussed, those algorithms are disabled because of the insufficiency on measurement noise in a harsh scenario. It is noted that the localization is influenced by choosing BAN. Therefore, generating proper LLS is a way to obtain the optimal localization accuracy instead of measurement error estimation.

The following section discusses the upper bound of the localization error propagation. We use the boundary to guide the construction of LLS which can be used in distribution infrastructure-free localization algorithm.

\section{Localization error upper boundary of anchors with errors}

One character of iterative localization is that the localization error propagates. We deduce an upper bound of the localization error propagation based on orthogonally invariant norms. In addition, an algorithm is proposed by using the upper bound as a LLS measurement.

\subsection{Orthogonally invariant norms}

The orthogonally invariant norms are used to conduct the upper bound of the localization error propagation. The notion and its characters are introduced as follows.
Definition 1 (Orthogonally Invariant Norms (Watson et al., [24]). Consider SVD of a given matrix $A, A$ have singular value decomposition

$$
A=U \Sigma V^{\mathrm{T}}
$$

where $U$ and $V$ are orthogonal matrices and $\Sigma$ is an $m \times n$ diagonal matrix, where the diagonal terms are the singular values of $A$ in descending order

$$
\sigma_{1} \geq \sigma_{2} \cdots \geq \sigma_{n}
$$

Orthogonally invariant norms can be defined by

$$
\|A\|=\phi(\sigma)
$$

where $\sigma=\left(\sigma_{1}, \cdots, \sigma_{n}\right)^{T}$ and $\phi$ is a symmetric gauge function, such a function satisfies the following conditions:

(1) $\Phi(x)>0, x \neq 0$,

(2) $\Phi(\alpha x)=|\alpha| \Phi(x), \forall \alpha \in \mathbb{R}$,

(3) $\Phi(x+y) \leqslant \Phi(x)+\Phi(y)$

(4) $\Phi\left(\varepsilon_{1} x_{i_{1}}, \cdots, \varepsilon_{n} x_{i_{n}}\right)=\Phi(x)$,

where $\alpha$ is a scalar, $\varepsilon_{i}= \pm 1$ for all $i$, and $i_{1}, \cdots, i_{n}$ is a permutation of $1,2, \cdots, n$.

Also, the following characters of the norm, which will be used in the Section 4.2, are obtained:

(1) $\left\|A^{\mathrm{T}}\right\|=\|A\|, \forall A \in \mathbb{C}^{m \times n}$

(2) $\|x\|=\|x\|_{2}, \forall x \in \mathbb{C}^{n}$

(3) $\|A B\| \leqslant\|A\|_{2}\|B\|, \forall A \in \mathbb{C}^{m \times n}, \forall B \in \mathbb{C}^{n \times l}$

(4) $\|A B\| \leqslant\|A\|\|B\|_{2}, \forall A \in \mathbb{C}^{m \times n}, \forall B \in \mathbb{C}^{n \times l}$

(5) $\|A-B\| \leq\|A\|+\|B\|, \forall A, B \in \mathbb{C}^{m \times n}$

(6) $\|A\|-\|B\| \mid \leqslant\|A-B\|$

4.2 Upper boundary of localization error of LLS-RSS iterative algorithm

4.2.1 Upper boundary of localization of error LLS

We propose a lemma which describes the upper bound of localization error for a LLS. The lemma issues an abstract but a useful formula for calculating the upper bound of the error propagation.

Theorem 1. Assuming ith anchor node is chosen as the $B A N$, a LLS is expressed as

$$
\hat{A}_{i} \hat{\boldsymbol{x}}_{i}^{\mathrm{T}}=\hat{\boldsymbol{b}}_{i}
$$

where $\hat{A}_{i}=A_{i}+\Delta \hat{A}_{i}$ is a matrix constructed by anchors' positions, $A_{i}$ represents precise physical position of anchor nodes, $\Delta \hat{A}_{i}$ is the coordinate errors of the anchors; $\hat{\boldsymbol{b}}_{i}=\boldsymbol{b}_{i}+\Delta \hat{\boldsymbol{b}}_{i}$ is a vector collection of the anchors' position and the measurement data, $\boldsymbol{b}_{i}$ denotes the noiseless measurement data, $\Delta \hat{\boldsymbol{b}}_{i}$ represents the noise of the measurement data. The ratio of localization coordinate to physical coordinate satisfies 


$$
\frac{\left\|\hat{\boldsymbol{x}}_{i}\right\|}{\|\boldsymbol{x}\|} \leqslant \kappa(1+\alpha)(1+\beta)
$$

where

$$
\begin{aligned}
& \kappa=\left\|\hat{A}_{i}^{\dagger}\right\|\left\|\hat{A}_{i}\right\| \\
& \alpha=\frac{\left\|\Delta \hat{A}_{i}\right\|_{2}}{\left\|\hat{A}_{i}\right\|_{2}} \\
& \beta=\frac{1}{\left\|\hat{\boldsymbol{b}}_{i}\right\|_{2} /\left\|\Delta \hat{\boldsymbol{b}}_{i}\right\|_{2}-1 \mid}
\end{aligned}
$$

Proof. First, based on the LLS expression $\hat{A}_{i} \hat{\boldsymbol{x}}_{i}=\boldsymbol{b}_{i}+\Delta$ $\hat{\boldsymbol{b}}_{i}, \hat{\boldsymbol{x}}_{i}$ is calculated as

$$
\hat{\boldsymbol{x}}_{i}=\hat{A}_{i}^{\dagger} \boldsymbol{b}_{i}+\hat{A}^{\dagger}{ }_{i} \Delta \hat{\boldsymbol{b}}_{i}
$$

where $\hat{A}_{i}^{+}$is Moore-Penrose pseudo-inverse of matrix $\hat{A}_{i}$.

Then, applying norm characters on (12), an inequality is obtained

$$
\left\|\hat{\boldsymbol{x}}_{i}^{\mathrm{T}}\right\| \leqslant\left\|\hat{A}_{i}^{\dagger}\right\|_{2}\left\|\boldsymbol{b}_{i}\right\|_{2}+\left\|\hat{A}^{\dagger}{ }_{i}\right\|_{2}\left\|\Delta \hat{\boldsymbol{b}}_{i}\right\|_{2}
$$

The inequality can be transformed into

$$
\frac{\left\|\hat{\boldsymbol{x}}_{i}\right\|}{\|\boldsymbol{x}\|} \leqslant\left\|\hat{A}^{\dagger}{ }_{i}\right\|_{2} \frac{\left\|\boldsymbol{b}_{i}\right\|_{2}}{\|\boldsymbol{x}\|}+\left\|\hat{A}^{\dagger}{ }_{i}\right\|_{2} \frac{\left\|\Delta \hat{\boldsymbol{b}}_{i}\right\|_{2}}{\|\boldsymbol{x}\|}
$$

It is noted that $A_{i} \boldsymbol{x}_{i}^{\mathrm{T}}=\boldsymbol{b}_{i}$. Inequalities

$$
\left\{\begin{array}{c}
\left\|\boldsymbol{b}_{i}\right\| \leqslant\left\|A_{i}\right\|_{2}\|\boldsymbol{x}\|_{2} \\
1 /\|\boldsymbol{x}\| \leqslant\left\|A_{i}\right\|_{2} /\|\boldsymbol{b}\|_{2}
\end{array}\right.
$$

are concluded. Using those inequalities, (14) becomes

$$
\frac{\left\|\hat{\boldsymbol{x}}_{i}\right\|}{\|\boldsymbol{x}\|} \leqslant\left\|\hat{A}^{\dagger}{ }_{i}\right\|_{2}\left\|\hat{A}^{\dagger}{ }_{i}\right\|_{2}\left(1+\frac{\left\|\Delta \hat{\boldsymbol{b}}_{i}\right\|_{2}}{\left\|\boldsymbol{b}_{i}\right\|_{2}}\right)
$$

For $\left\|\hat{b}_{i}\right\|_{2}-\left\|\hat{b}_{i}-\Delta \hat{b}_{i}\right\|_{2}$, it is $\left\|\hat{b}_{i}\right\|_{2} \geq\left|\left\|\hat{b}_{i}-\Delta \hat{b}_{i}\right\|_{2}\right|$. Therefore,

$$
\frac{\left\|\hat{\boldsymbol{x}}_{i}\right\|}{\|\boldsymbol{x}\|} \leqslant\left\|\hat{A}^{\dagger}{ }_{i}\right\|_{2}\left\|A_{i}\right\|_{2}(1+\beta)
$$

where $\beta=\left|\left\|\hat{\boldsymbol{b}}_{i}\right\|_{2} /\left\|\Delta \hat{\boldsymbol{b}}_{i}\right\|_{2}-1\right|^{-1}$.

Because of $\left\|A_{i}\right\|_{2}=\left\|\hat{A}_{i}-\left(\hat{A}_{i}-A_{i}\right)\right\|_{2}$, it is obtained that

$$
\left\|A_{i}\right\|_{2} \leqslant\left\|\hat{A}_{i}\right\|_{2}+\left\|\Delta \hat{A}_{i}\right\|_{2}
$$

Thus,

$$
\left\|\hat{A}_{i}^{\dagger}\right\|_{2}\left\|A_{i}\right\|_{2} \leq \kappa\left(1+\left\|\Delta \hat{A}_{i}\right\|_{2} /\left\|\hat{A}_{i}\right\|_{2}\right)
$$

where $\kappa=\left\|\hat{A}_{i}^{\dagger}\right\|\left\|\hat{A}_{i}\right\|$. Finally, combining (17) and (19), the conclusion is obtained.

\subsubsection{Error upper boundary of LLS-RSS}

The Theorem 1 gives a universal upper bound of the measurement error. Since RSS is widely used as the measurement data, we propose a concrete numeral upper bound of the measurement error of a LLS-based RSS (LLS-RSS). The upper bound will be fundamental of algorithm which can construct the optimum LLS in the next subsection.

To calculate the upper bound of Lemma 1, we need to calculate $k, \alpha$, and $\beta$. The $k$ and $\alpha$ are calculable, because all components are only related with known coordinate of anchors (origin-anchors, pseudo-anchors, or combination of them). However, the measurement data noise $\Delta \hat{\boldsymbol{b}}_{i}$ is random and unmeasurable. It makes instantaneous value of $\beta$ that cannot be calculated. The value can be obtained is the mean of $\beta$ which is upper boundary. As an extension of Theorem 1, the mean of error upper bound of LLS-RSS is given by Lemma 2 .

Theorem 2. In a LLS-RSS expressed as $\hat{A}_{i} \hat{\boldsymbol{x}}_{i}^{\mathrm{T}}=\hat{\boldsymbol{b}}_{i}+\Delta$ $\hat{\boldsymbol{b}}_{i}, \Delta \hat{A}_{i}$ is a matrix constructed by the minimum upper bound of localization error of corresponding anchors. If the radio propagation model between ith node and $k$ th node satisfies the model of distance-dependent path loss with log-normal fading, whose parameters are $\eta$ and $X_{\sigma_{i}}$, and random variable $X_{\sigma_{i}}(i=1, \cdots, n-1)$ are independent and identically distributed, there is

$$
E\left[\frac{\left\|\hat{x}_{i}\right\|}{\|x\|}\right] \leq \kappa(1+\varsigma)\left(1+\frac{1}{\xi-1}\right)
$$

where

$$
\begin{aligned}
& \varsigma=\frac{\sqrt{\sum_{k=1(k \neq i)}^{n}\left\|\Delta \boldsymbol{x}_{k}\right\|_{2}^{2}}+(n-1)\left\|\Delta \boldsymbol{x}_{k}\right\|_{2}^{2}}{\sqrt{\sum_{k=1(k \neq i)}^{n}\left\|\boldsymbol{x}_{k}-\boldsymbol{x}_{i}\right\|_{2}^{2}}} \\
& \xi=\frac{\sqrt{\sum_{k=1(k \neq i)}^{n}\left(\left\|\boldsymbol{x}_{k}\right\|_{2}^{2}-\hat{d}_{k}^{2}-\left\|\boldsymbol{x}_{i}\right\|_{2}^{2}+\hat{d}_{i}^{2}\right)^{2}}}{|c| \sqrt{\sum_{k=1, k \neq i}^{n}\left(\hat{d}_{i}^{2}-\hat{d}_{k}^{2}\right)^{2}}+\sqrt{\sum_{k=1(k \neq i)}^{n}\left\|\Delta \boldsymbol{x}_{k}\right\|_{2}^{2}}+(n-1)\left\|\Delta \boldsymbol{x}_{k}\right\|_{2}^{2}} \\
& c=1-\exp \left[\frac{1}{2}\left(\frac{2 \ln (10)}{10 \eta} \sigma\right)^{2}\right]
\end{aligned}
$$

Proof. According to the radio propagation model of distance-dependent path loss with lognormal fading [25], we have:

$$
P_{r}\left(d_{r}\right)=P_{0}-\eta 10 \log _{10}\left(\frac{d_{i}}{d_{0}}\right)+X_{\sigma_{i}}
$$

The distance between $i$ th anchor and the unknown node, denoted as $d_{i}$, should be calculated as

$$
d_{k}=d_{0} 10^{\frac{P_{0}-P_{r}(d r)+X_{\sigma_{i}}}{10 \eta}}
$$


However, since $X_{\sigma_{i}}$ is a physically immeasurable random variable, the $k$ th estimated distance, denoted as $\hat{d}_{k}$ is calculated as

$$
\hat{d}_{k}=d_{0} 10^{\frac{P_{0}-P_{r}\left(d_{r}\right)}{10 \eta}}
$$

Introduce a $\Delta d^{2}$, which is defined as

$$
\Delta \hat{d}_{k}^{2} \triangleq \hat{d}_{k}^{2}-d_{k}^{2}=\hat{d}_{k}^{2}\left(1-10^{\frac{2 X_{\sigma_{i}}}{10 \eta}}\right)
$$

The mean of $\Delta d^{2}$ is known as [3]

$$
E\left[\Delta \hat{d}_{k}^{2}\right]=\hat{d}_{k}^{2}\left(1-\exp \frac{1}{2}\left(\frac{2 \ln (10)}{10 \eta} \sigma\right)^{2}\right)
$$

Consider the definition of $b_{k}^{i}$, $k$ th component of $\mathrm{b}_{i}$, then the $b_{k}^{i}$ can be donated as

$$
b_{k}^{i}=\left\|\boldsymbol{x}_{k}\right\|_{2}^{2}+\hat{d}_{k}^{2}-\left(\left\|\boldsymbol{x}_{i}\right\|_{2}^{2}-\hat{d}_{i}^{2}\right)
$$

In a practical localization system, the noise always exists in measurement data. It is defined as

$$
b_{k}^{i} \triangleq \hat{b}_{k}^{i}-\left(\Delta \hat{b}_{k, 1}^{i}+\Delta \hat{b}_{k, 2}^{i}\right)
$$

We introduce $\Delta \hat{b}_{i, 1}$ and $\Delta \hat{b}_{i, 2}$ to denote the vectors whose elements are $\hat{b}_{i, 1}$ and $\hat{b}_{i, 2}$, respectively. Thus, there is

$$
E\left(\frac{\left\|\hat{\boldsymbol{b}}_{i}\right\|_{2}}{\left\|\Delta \hat{\boldsymbol{b}}_{i}\right\|_{2}}\right) \leqslant \frac{\left\|\hat{\boldsymbol{b}}_{i}\right\|_{2}}{\left\|E\left(\Delta \hat{b}_{k, 1}^{i}\right)\right\|_{2}+\left\|E\left(\Delta \hat{b}_{k, 2}^{i}\right)\right\|_{2}}
$$

where $\left\|E\left(\Delta \hat{\boldsymbol{b}}_{i, 2}\right)\right\|_{2}=\left\|E\left(\Delta \hat{d}_{i}\right)-E\left(\Delta \hat{d}_{k}\right)\right\|$ since the random variables are independent and identically distributed.

Use Theorem 1, Combine (25), (29) and norm definition, the (20) and (21) are obtained.

\subsection{Optimum algorithm of constructing LLS}

Theorem 2 gives the numerical result of localization accuracy influenced by node information and measurement data. The theorem can handle the situation that the positions of the anchor nodes can exist error. Although the error upper bound is calculated in statistical significance, $\Delta b_{\mathrm{i}} / b_{\mathrm{i}}$ appears with a high probability, which is tested in experiments.

Therefore, the minimum upper bound can be used as a localization quality indicator of the LLS. An LLS construction algorithm, optimum algorithm of constructing LLS (OAC-LLS) shown as Algorithm 1, is proposed. This algorithm utilizes the minimum upper bound to choose the best candidate from the LLS set with a high probability.

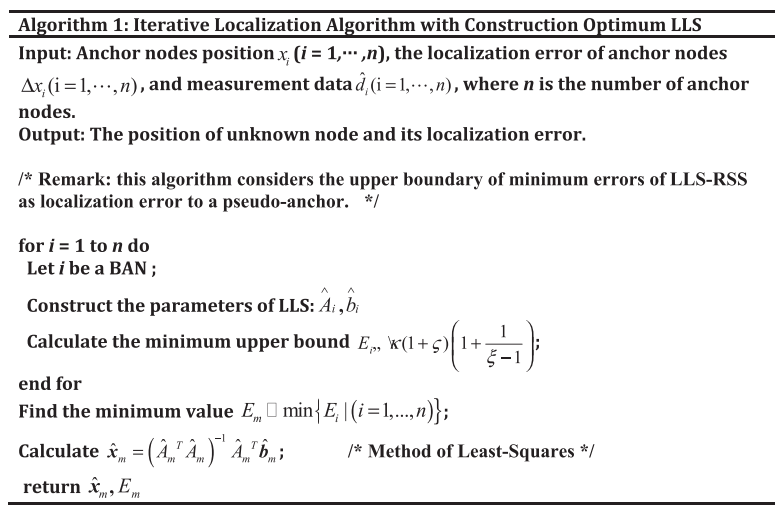

Remark: The environment parameter $\sigma / \eta$ is needed to calculate the $E_{i}$ in (19). But, a estimated value can be used. It will not significantly affect the result. It means that the algorithm could be fully "blind" based on an assumption value. Of course, any knowledge of parameter can improve the algorithm performance. This conclusion is discussed in Section 5.2.

\section{Simulation and discussion}

The following assumptions are used in experiments.

(i) We use three origin-anchor nodes, whose coordinates are $(0,0),(50,0)$, and $(25,50)$, respectively. The nodes are numbered by their orders. The fourth node is a pseudo-anchor whose coordinate is $(25,25)$. The fifth node is a pseudo-anchor.

(ii) The radio propagation model uses distance-dependent path loss with log-normal fading with Gaussian noise $\mathrm{N}(0,1.5)$.

(iii) The position of the unknown node is calculated as

$$
\hat{\boldsymbol{x}}_{m}=\left(\hat{A}_{m}^{\mathrm{T}} \hat{A}_{m}\right)^{-1} \hat{A}_{m}^{\mathrm{T}} \boldsymbol{b}_{m} \text {. }
$$

To distinguish the different LLS, which use different anchor set of origin-anchors or pseudo-anchors, we add a superscript on $\hat{A}_{i}$ to declare the used anchor nodes. Therefore, $\hat{A}_{1}^{1-2-3}$ means the LLS uses three nodes whose numbers are 1,2 , and 3 , and 1-st node are chosen as BAN.

\subsection{Evaluation indicator}

The fundamental of algorithm is the mean of error upper bound. It is noted that the upper bound is given in statistical perspective. It is possible that the upper bound is minimum while the localization is not optimum. The evaluation indicator of algorithm should reflect that the algorithm can choose optimum LLS or not using upper 


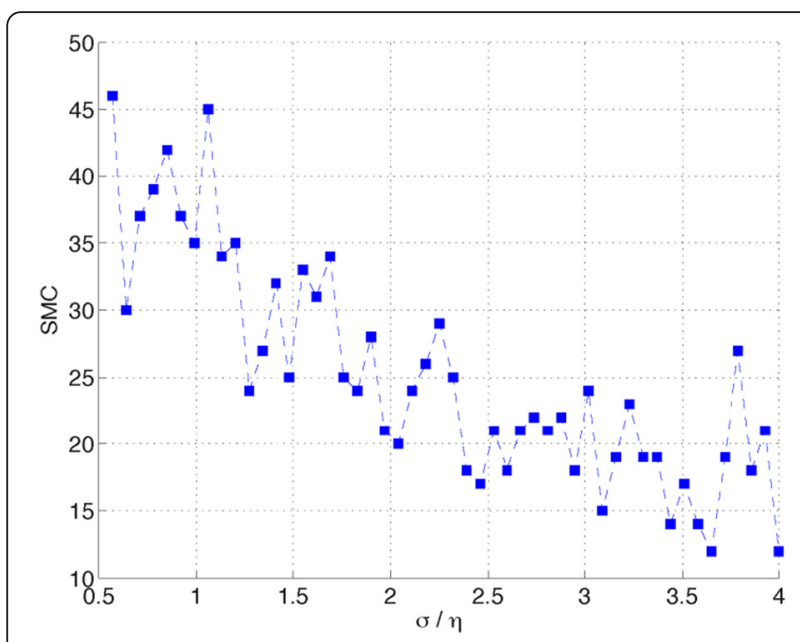

Fig. 1 Experiments result of probability of construction optimum LLS varies with $\sigma / \eta$

bound. Therefore, the coordinate error is unsuitable to evaluate the algorithm performance.

To evaluate the efficiency of improving accuracy using minimum rough upper bound, we introduce two counters:

(a) strict-match-counter (SMC) and (b) slack-match-count (LMC). The counters work as following:

- SMC: Let initial value of SMC be 0. SMC increases 1 , if and only if, the minimum of rough upper bound and the minimum of localization absolute error are both obtained when $i$ th anchor is selected as a BAN.

- LMC: It is similar to SMC. However, LMC increases 1 when localization absolute error is smallest or

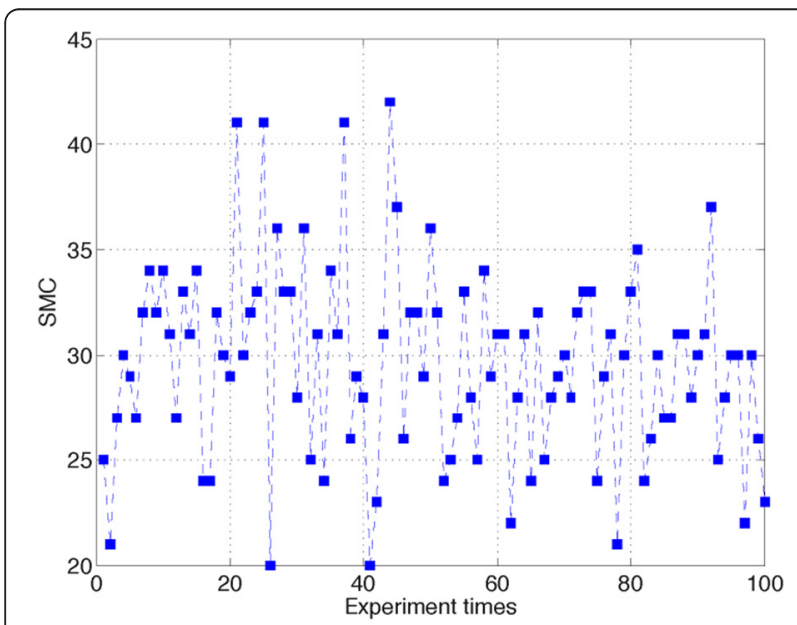

Fig. 2 Experiments result that the minimum upper bound matches with optimum LLS. The simulation is done $100 \times 100$ times, and each value is the match count per 100 times simulations using SMC

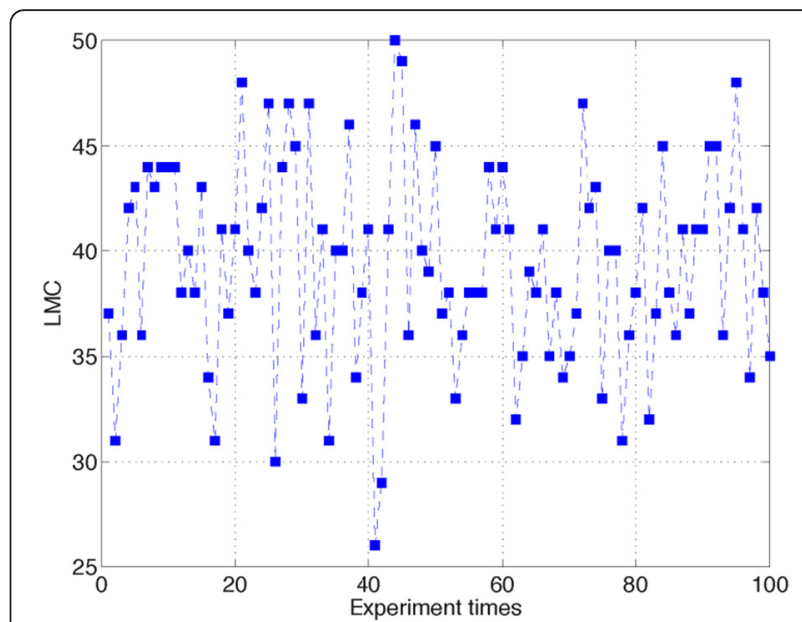

Fig. 3 Experiments result that the minimum upper bound matches with optimum LLS. The simulation is done $100 \times 100$ times, and each value is the match count per 100 times simulations using LMC

second smallest in the case of the upper bound is minimum with the same BAN.

\subsection{Feasibility of the algorithm}

Each value is the match count per 100 times simulations using SMC. The calculation of minimum upper bound uses $\sigma / \eta=0.65$ in each time.

To calculate the upper bound by using Theorem $2, \sigma / \eta$ is needed to obtain $c$. Experiments are implemented to illustrate the probability of construction optimum LLS varies with $\sigma / \eta$. The fifth node is placed at $(25,6), \sigma / \eta=0.65$ is used in each time of calculation of minimum upper bound.

As shown in Fig. 1, the probability of construction optimum LLS varies with $\sigma / \eta$. The curve shows a

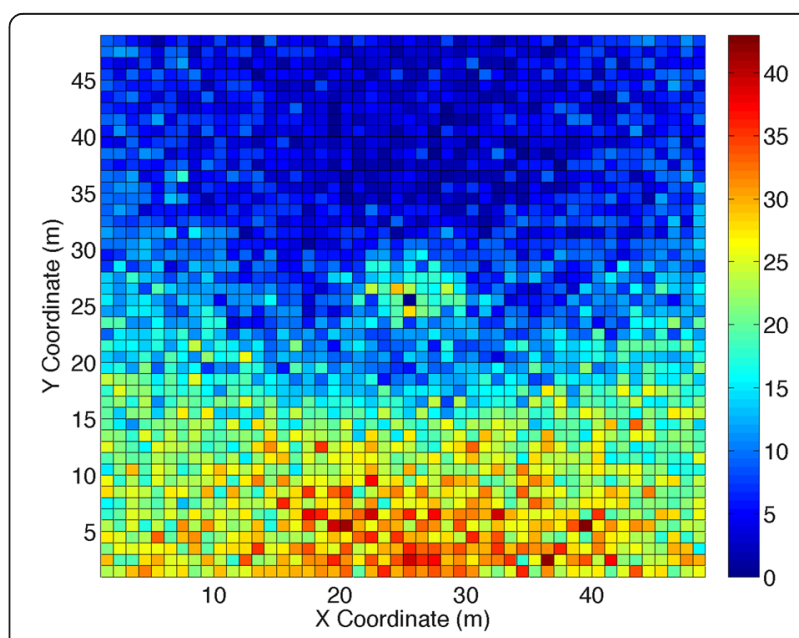

Fig. 4 Each colored block represents the matching counts per 100 times using SMC, when the fifth node is fixed at a certain coordinate. The noise is random in each time. The green starts are origin-anchors and pseudo-anchor used to locate 5 -th node 


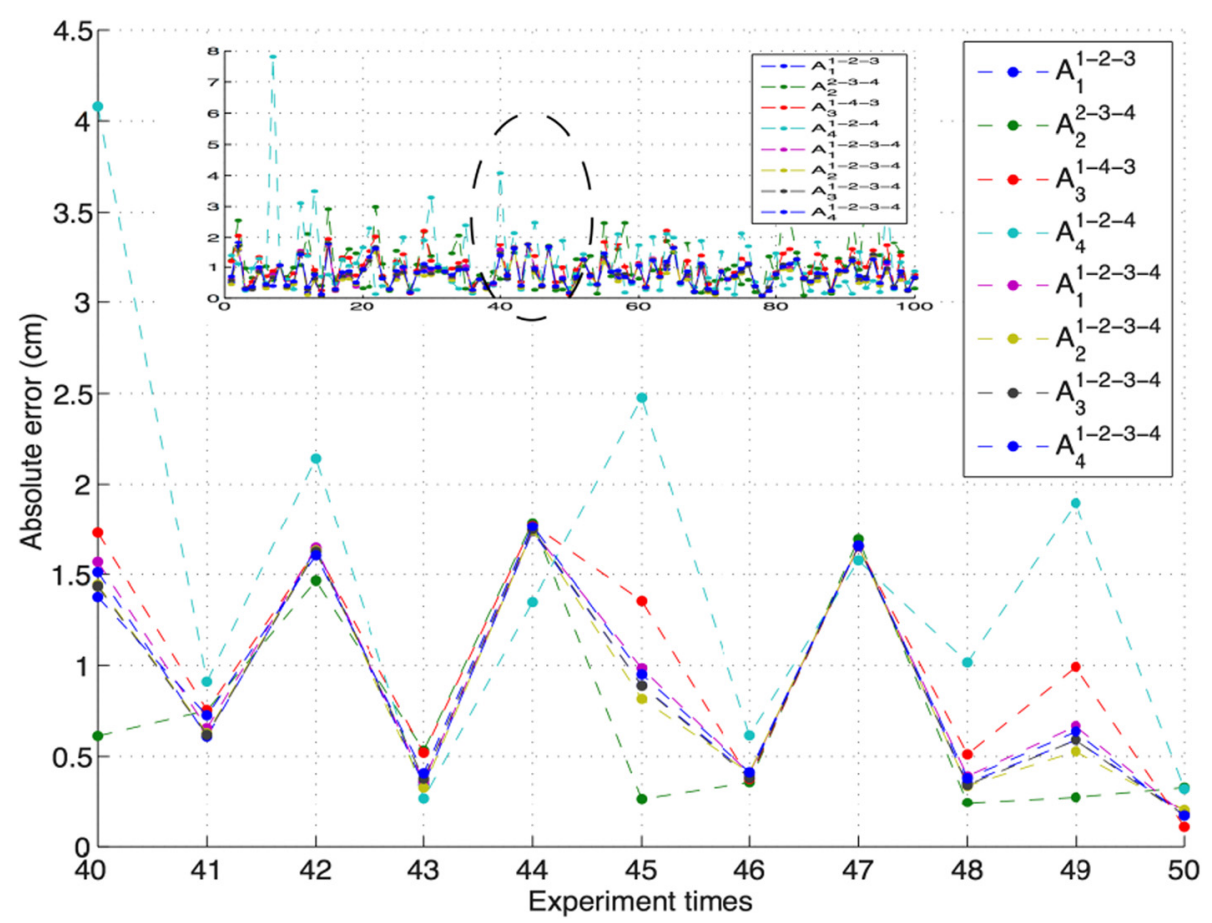

Fig. 5 Localization accuracies vary with different BANs. The unknown node is fixed at $(25,45)$, and noise is random in each time

trend that the probability is descent while the $\sigma / \eta$ is away from the precise value. It means that the algorithm could be fully "blind" on the environment. Meanwhile, any information of environment, such as the possible range of $\sigma / \eta$, can greatly improve the algorithm performance.

\subsection{Randomness of the algorithm}

Experiments are executed to test the randomness of the algorithm. The fifth node is placed at $(25,6)$, and the experiments are executed for $100 \times 100$ times.

As shown in Fig. 2, if the SMC is selected as an evaluation indicator, the mean and standard deviation of

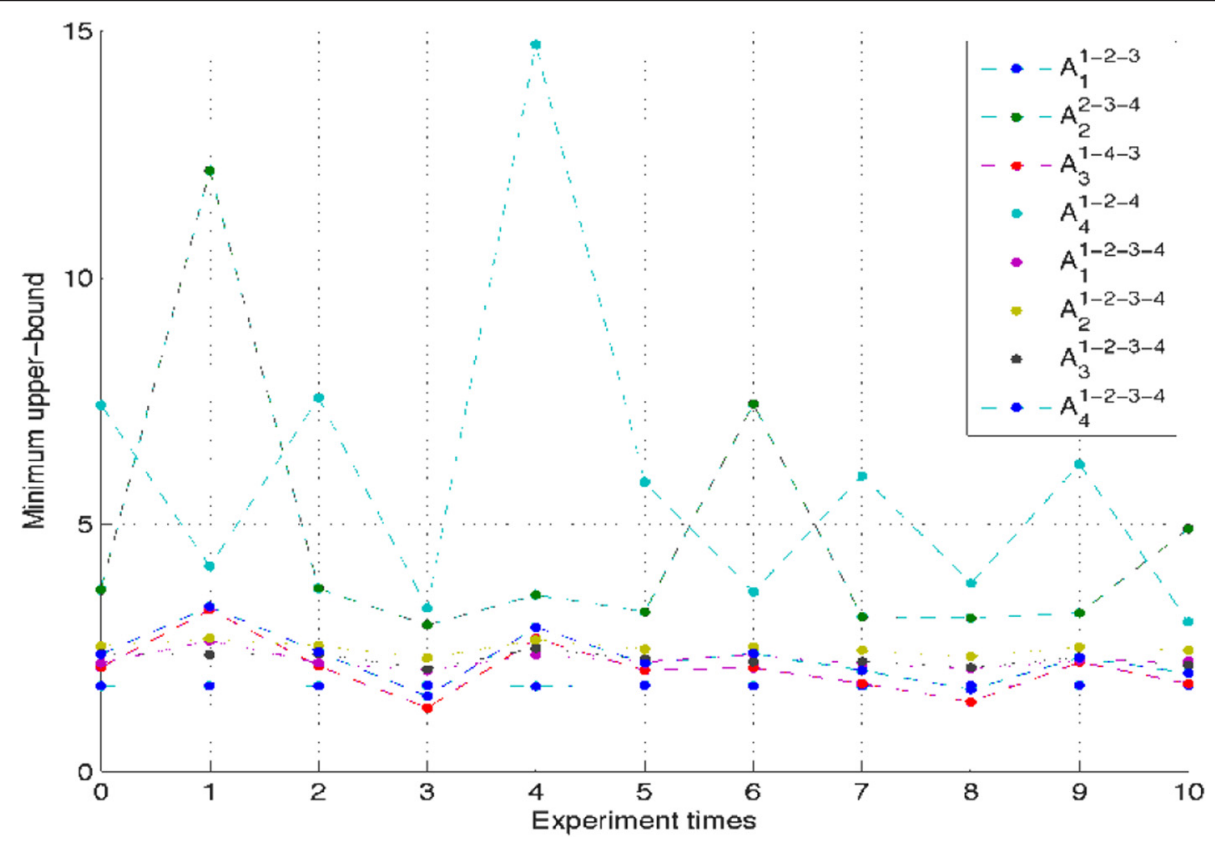

Fig. 6 Minimum error upper bounds vary with different BANs. Each upper bound corresponds each localization accuracy in Fig. 5 


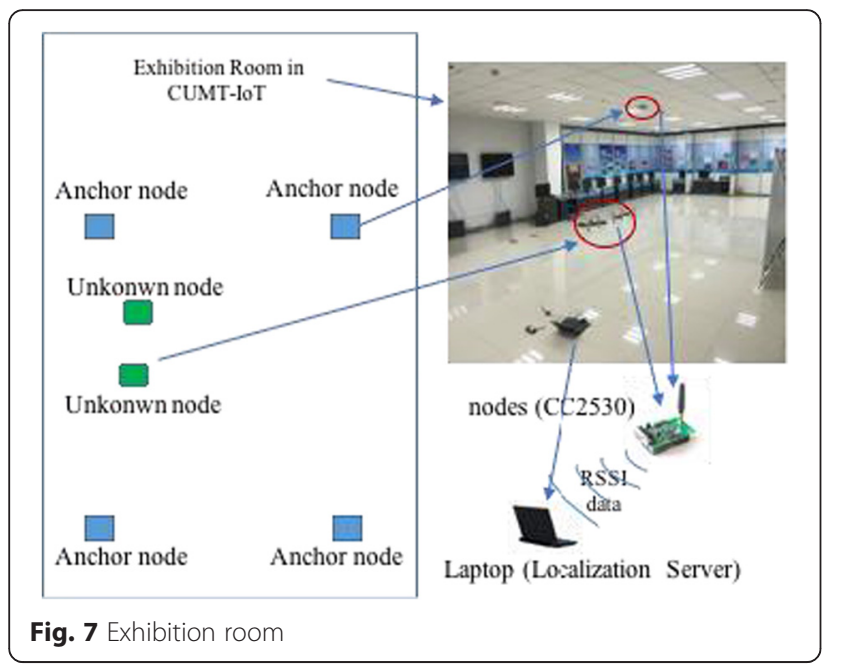

probability of choosing best LLS are 0.2959 and 4.57 , respectively. In the same way, shown in Fig. 3, if LMC is used as an evaluation indicator, the mean and standard deviation of probability of constructing best LLS are 0.3934 and 4.86 , respectively. It is noted that there are totally eight LLS can be constructed. The probability choosing optimum LLS is 0.125 . Our algorithm can generate the best LLS with a higher probability.

\subsection{Effectiveness of the algorithm}

Figure 4 shows the algorithm effectiveness varies with change of the pseudo-anchor's position. The algorithm is more effective when the fifth node in the triangle zone bordered by the first, third, and fourth nodes. While the fifth node moves away the zone, the probability of constructing optimum LLS deceases. This phenomenon is reasonable. The localization accuracy of fourth node is stable and precise since the qualities of measurement data are almost the same (Yang et al., [26]). It means that the fifth node has an additional pseudo-anchor with high accuracy besides four origin-anchors, which make the upper boundary more valuable.

Although the algorithm does not have a good numerical performance when the SMC is used as indicator, it is noted that the worst accuracy will not be obtained, as shown in Figs. 5 and 6. In fact, the localization accuracies are tightly close when the minimum upper bounds are approximately the same, which is shown as enlarged party of Figs. 5 and 6 . Therefore, the algorithm is still effective in the perspective of improving localization accuracy.

Additionally, Figs. 5 and 6 show that the curve of absolute errors gently changes when the LLS is the one with minimum upper boundary. It means that the algorithm is stable. The factor of upper bound, $\kappa=\left\|\hat{A}_{m}\right\|_{2}\left\|\hat{A}_{m}\right\|_{2}$, is a condition number, which means that the condition number of LLS will be smaller when the upper bound is minimum. Therefore, the algorithm, which uses minimum upper bound, is insensitive to noise.

\subsection{Performance evaluation}

Experiments are done in our exhibition room. The scenario is showed as in Fig. 7. All nodes are based on CC2530. Four of them, which are considered as anchor nodes, are fixed on the ceiling. The others are considered as unknown nodes. A laptop is used to sample data and servers as localization server.

Figure 8 illustrates the cumulative distribution function (CDF) of localization errors. The experiment results show that the error falls within the range of $2 \mathrm{~m}$ for over $90 \%$ of points, and the $50 \%$ accuracy is less than $1.2 \mathrm{~m}$. The algorithm 1 has a better performance than the

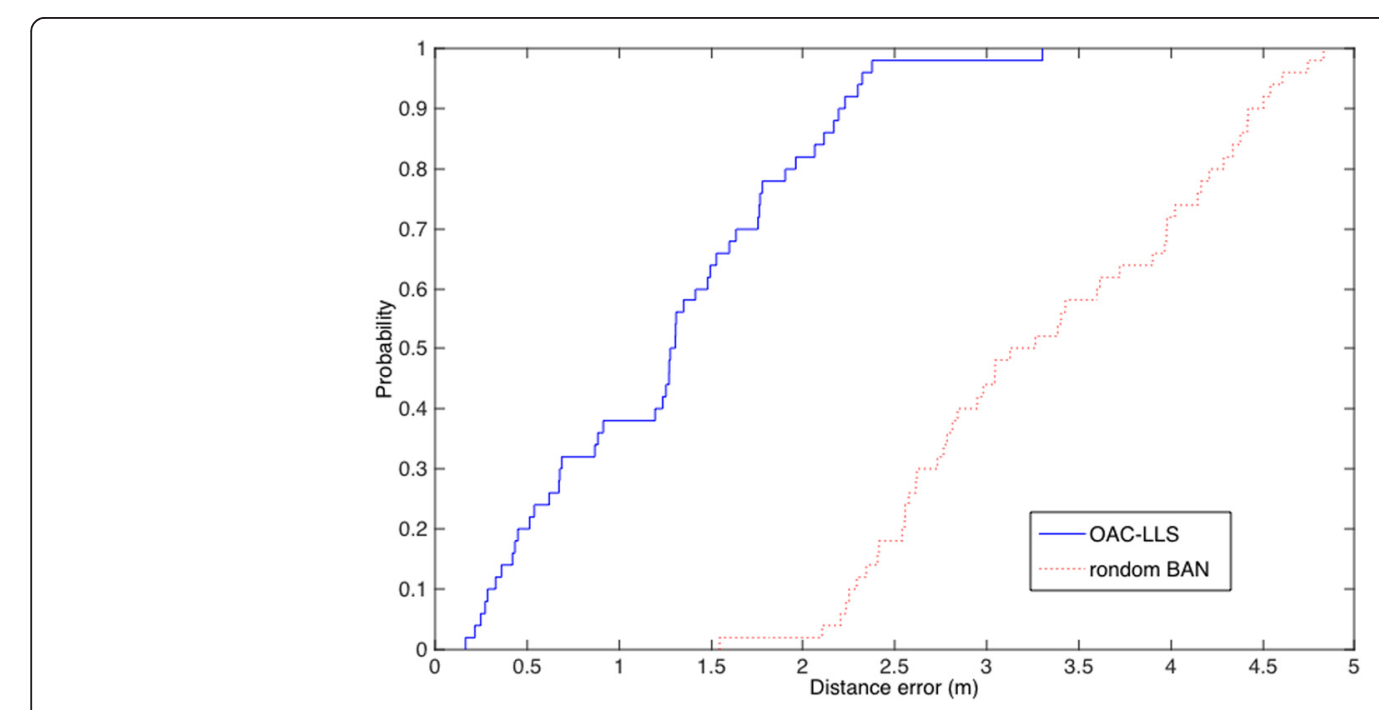

Fig. 8 CDF of localization error in exhibition room 
algorithm which BAN is chosen random. The probability choosing optimum LLS is 0.125 since there are totally eight LLS that can be constructed. However, this probability can approach 0.45 as simulated in Section 5.3. Therefore, we can obtain more accurate coordinate compared to the coordinate obtained when BAN is random selected.

\subsection{Computational complex}

In some application of mobile autonomous robots, the energy consumption and computation ability are constrained. It requires the localization to be realized on-chip. Computational complexity, which is defined as the number of operations performed by the algorithm [27], is always used to evaluate the possibility implemented on chip.

Algorithm 1 consists of a choosing BAN process and a least-squares algorithm. Assume the algorithm is used in 2D localization, in which case $x_{i}, \Delta x_{i}(i=1, \cdots, n)$ are two dimensional vectors. The time complexity of leastsquares algorithm will be $O\left(n^{3}\right)$. To find the best BAN, we first execute $n$ loops to obtain $E_{i}(i=1, \cdots, n)$, then we find the minimum $E_{m}$ which is the best BAN. It is noted that $\left\|\hat{A}_{i}^{\dagger}\right\|_{2},\left\|\hat{A}_{i}\right\|_{2},\left\|\Delta \boldsymbol{x}_{k}\right\|_{2}^{2},\left\|\boldsymbol{x}_{k}-\boldsymbol{x}_{i}\right\|_{2}^{2}, \hat{d}_{k}^{2}$, and $\left(\hat{d}_{i}^{2}-\hat{d}_{k}^{2}\right)^{2}$ are repeated used in algorithm. The time complexity of $\left\|\hat{A}_{i}^{\dagger}\right\|$ is $64 n+96+O(2 n)$ when using Matlab pinv function. The cost functions of other terms are shown as Table 1.

Therefore, the time complexity of algorithm 1 is $O\left(3 n^{3}\right)$. The algorithm is more complex than the least-squares algorithm. It is reasonable because the algorithm trades computational complex off for localization accuracy. It is also noticed that $n$ is the anchor node number, which is a small value in practice.

\section{Conclusions}

An upper bound of error propagation of iterative localization is derived, which can be used in the situation that the precise distribution of the environment noise is unknown. The minimum upper bound is adopted to evaluate the localization result of LLS with certain measurement data. With this method, an optimum algorithm of constructing LLS is proposed. Even when the environment noise is unknown or unpriced evaluated, the

Table 1 Cost functions

\begin{tabular}{lll}
\hline Title & Add & MUL \\
\hline$\left\|\hat{A}_{i}\right\|_{2}(i=1, \cdots, n)$ & $n(n-1)$ & $n^{2}$ \\
$\left\|\Delta \boldsymbol{x}_{i}\right\|_{2}^{2}(i=1, \cdots, n)$ & $(n-1)$ & $n$ \\
$\left\|\boldsymbol{x}_{k}-\boldsymbol{x}_{i}\right\|_{2}^{2}(i, k=1, \cdots, n ; k \neq i)$ & $n(n-1)$ & $(n-1)^{2}(n-2)$ \\
$\hat{d}_{i}^{2}(i=1, \cdots, n)$ & $n$ & $n$ \\
$\left(\hat{d}_{i}^{2}-\hat{d}_{k}^{2}\right)^{2}(i, k=1, \cdots, n ; k \neq i)$ & $n-1$ & $(n-1)^{2}(n-2)$ \\
\hline
\end{tabular}

algorithm still can construct the proper LLS with highly probability, which means it can still obtain the best localization accuracy with high probability.

\section{Acknowledgements}

This work is supported partly by the National Key Technology Research and Development Program of the Ministry of Science and Technology of China under grant (no: 2013BAK06B05) and the National Natural Science Foundation of China under grant (no: 61303183).

\section{Competing interests}

The author declares that he has no competing interests.

\section{Author details}

${ }^{1}$ IoT Perception Mine Research Center, China University of Mining and Technology, Xuzhou, People's Republic of China. ${ }^{2}$ School of Electrical Engineering and Automation, Jiangsu Normal University, Xuzhou, People's Republic of China. ${ }^{3}$ School of Information and Electrical Engineering, China University of Mining and Technology, Xuzhou, People's Republic of China.

Received: 13 March 2016 Accepted: 14 June 2016

Published online: 29 June 2016

References

1. J Fink, A Ribeiro, V Kumar, Robust control for mobility and wireless communication in cyber-physical systems with application to robot teams. Proc IEEE 100(1), 164-178 (2012)

2. L Ojeda, D Cruz, G Reina, J Borenstein, Current-based slippage detection and odometry correction for mobile robots and planetary rovers. Robotics, IEEE Transactions on 22(2), 366-378 (2006)

3. SS Saad, ZS Nakad, A standalone RFID indoor positioning system using passive tags. IEEE Trans Ind Electron 58(5), 1961-1970 (2011)

4. X Li, Collaborative localization with received signal strength in wireless sensor networks. Vehicular Technology, IEEE Transactions on 56(6), 3807-3817 (2007)

5. Z Z Ma, W Chen, KB Letaief, Z Cao, A semi range-based iterative localization algorithm for cognitive radio networks. Vehicular Technology, IEEE Transactions on 59(2), 704-717 (2010)

6. G Wang, K Yang, A new approach to sensor node localization using RSS measurements in wireless sensor networks. Wireless Communications, IEEE Transactions on 10(5), 1389-1395 (2011)

7. MR Gholami, EG Ström, H Wymeersch, Upper bounds on position error of a single location estimate in wireless sensor networks. Eurasip Journal on Advances in Signal Processing 1, 1-14 (2014)

8. P Dhakal, D Riviello, F Penna, Impact of noise estimation on energy detection and eigenvalue based spectrum sensing algorithms. IEEE International Conference on Communications IEEE, 1367-1372 (2014)

9. S Čapkun, M Hamdi, JP Hubaux, Gps-free positioning in mobile ad hoc networks. Clust Comput 5(2), 157-167 (2002)

10. $\amalg$ Zamorano, L Nolte, AM Kadi, Z Jiang, Interactive intraoperative localization using an infrared-based system. Neurol Res 15(5), 290-298 (1993)

11. J Liu, Y Zhang, F Zhao, Robust distributed node localization with error management, in Proceedings of the 7th ACM international symposium on Mobile ad hoc networking and computing, 2006, pp. 250-261

12. E DiGiampaolo, F Martinelli, Mobile robot localization using the phase of passive UHF RFID signals. IEEE Trans Ind Electron 61(1), 365-376 (2014)

13. Y Ma, EC Kan, Accurate indoor ranging by broadband harmonic generation in passive NLTL backscatter tags. Microwave Theory and Techniques, IEEE Transactions on 62(5), 1249-1261 (2014)

14. Z Yang, Z Zhou, Y Liu, From RSSI to CSI: indoor localization via channel response. ACM Computing Surveys (CSUR) 46(2), 25 (2013)

15. A Savvides, H Park, MB Srivastava, The bits and flops of the $n$-hop multilateration primitive for node localization problems, in Proceedings of the 1st ACM international workshop on Wireless sensor networks and applications, 2002, pp. 112-121

16. IA Mantilla-Gaviria, M Leonardi, G Galati, Localization algorithms for multilateration (MLAT) systems in airport surface surveillance. Signal Image \& Video Processing 9(7), 1-10 (2014)

17. C Chang, A Sahai, Cramer-rao-type bounds for localization. EURASIP Journal on Applied Signal Processing 2006, 1-13 (2006)

18. EG Larsson, Cramer-Rao bound analysis of distributed positioning in sensor networks. Signal Processing Letters, IEEE 11(3), 334-337 (2004) 
19. RL Moses, D Krishnamurthy, RM Patterson, A self-localization method for wireless sensor networks. EURASIP Journal on Applied Signal Processing 2003(4), 348-358 (2003)

20. A Savvides, WL Garber, RL Moses, MB Srivastava, An analysis of error inducing parameters in multihop sensor node localization. Mobile Computing, IEEE Transactions on 4(6), 567-577 (2005)

21. J Koo, $\mathrm{H}$ Cha, Localizing WiFi access points using signal strength. Communications Letters, IEEE 15(2), 187-189 (2011)

22. Z. Xu et al. Crowdsourcing based description of urban emergency events using social media big data. IEEE Transactions on Cloud Computing,10. 1109/TCC.2016.2517638.

23. Z. Xu et al. Crowdsourcing based social media data analysis of urban emergency events. Multimedia Tools and Applications, 10.1007/s11042-015-2731-1.

24. GA Watson, Characterization of the subdifferential of some matrix norms. Linear Algebra Appl 170, 33-45 (1992)

25. M Shin, I Joe, An indoor localization system considering channel interference and the reliability of the RSSI measurement to enhance location accuracy. International Conference on Advanced Communication Technology IEEE, (2015)

26. Z Yang, Y Liu, Quality of trilateration: Confidence-based iterative localization. Parallel and Distributed Systems, IEEE Transactions on 21(5), 631-640 (2010)

27. M Sipser, Introduction to the Theory of Computation, vol. 2 (Thomson Course Technology, Boston, 2006)

\section{Submit your manuscript to a SpringerOpen ${ }^{\odot}$ journal and benefit from:}

- Convenient online submission

- Rigorous peer review

- Immediate publication on acceptance

- Open access: articles freely available online

- High visibility within the field

- Retaining the copyright to your article

Submit your next manuscript at $\boldsymbol{s p r i n g e r o p e n . c o m ~}$ 